\title{
Influencia de los sistemas de puesta a tierra descritos en el estándar IEEE 1243 en la tasa de fallas por rayos de líneas de transmisión Influence of the earthing systems described in the IEEE 1243 Standard on the back- flashover rate of transmission lines
}

\author{
Jaime Alejandro Mariño-Rojas ${ }^{1 a}$, Jonathan Armando Muñoz-Delgado ${ }^{1 b}$, \\ Edison Andrés Soto-Ríos ${ }^{1 c}$
}

\begin{abstract}
${ }^{1}$ Grupo de Investigación en Sistemas de Energía Eléctrica - GISEL, Universidad Industrial de Santander, Colombia. Correos electrónicos: ${ }^{\mathrm{a}}$ alejandromarinho11@gmail.com, ${ }^{\mathrm{b}}$ jonathan.munoz@correo.uis.edu.co, ${ }^{\mathrm{c}}$ easotor@uis.edu.co
\end{abstract}

Recibido: 20 enero, 2020. Aceptado: 11 julio, 2020. Versión final: 19 noviembre, 2020.

\begin{abstract}
Resumen
Este trabajo trata sobre los dos diferentes modelos resistivos de puesta a tierra usados en líneas de transmisión presentados en el estándar IEEE 1243-1997: tierra suplementaria con varillas y con contrapesos. Se analizó el efecto de variar los parámetros internos de cada modelo que afectan el valor resistivo de puesta a tierra en la reducción o aumento de la tasa de fallas por flameo inverso por impactos de rayos sobre la torre o el cable de guarda sobre terrenos con diferentes conductividades. El software ATPDraw se usó para obtener las corrientes críticas, para luego con estas obtener la tasa de fallas de la línea. Se simuló el efecto del número, la longitud, radio y enterramiento de los electrodos en las tasas de fallas por flameo inverso de una línea de transmisión para diferentes valores de conductividad del terreno. Se obtuvieron porcentajes de reducción de la tasa de fallas que van en general desde el 50 hasta el $95 \%$.
\end{abstract}

Palabras clave: BFR; descargas atmosféricas; ATPDraw; tasa de fallas; sistemas de puesta a tierra (SPT); IEEE 1243; líneas de transmisión.

\begin{abstract}
This work deals with two kind of resistive models for grounding in transmission lines portrayed in the IEEE 12431997 Standard: counterpoises and supplemental groundings rods. It is analyzed the effect of varying the internal parameters of each model that affect the resistive value of grounding in the reduction or the increase of the backflashover rate due to lightning strikes to the structure or to the ground wire over terrains with several conductivities. It is used the ATPDraw software to obtain the critical currents and then to obtain the back-flashover rate of the line. It was simulated the effect of the number, longitude, radius and depth of the electrodes in the back-flashover rate of a transmission line for several values of ground conductivity. It was obtained reductions percentages of the backflashover rate between 50 and $95 \%$.
\end{abstract}

Keywords: atmospheric discharges; ATPDraw; back-flashover rate; failure rate; grounding system; IEEE 1243; transmission lines.

ISSN impreso: 1657 - 4583. ISSN en línea: 2145 - 8456, CC BY-ND 4.0 @ (c) @

Como citar: J. Mariño, J. Muñoz, E. Soto, "Influencia de los sistemas de puesta a tierra descritos en el estándar IEEE 1243 en la tasa de fallas por rayos de líneas de transmisión," Rev. UIS Ing., vol. 20, no. 1, pp. 223-238, 2021, doi: 10.18273/revuin.v20n1-2021019 


\section{Introducción}

Las descargas atmosféricas son las principales causantes de fallas momentáneas o permanentes en líneas de transmisión, resultando en daños en los equipos conectados, y/o en el sistema eléctrico, generando cuantiosas pérdidas económicas [1]. Los reglamentos vigentes como el RETIE o el estándar IEEE 1243-1997 dan una pauta bajo la cual se diseñan los sistemas de puesta a tierra lo cual tiene un impacto importante en la reducción de fallas por descargas atmosféricas. Los rayos más comunes a nivel mundial son los negativos nubetierra, sin embargo, estudios previos hechos han encontrado que el más común en Colombia corresponde a rayos positivos nube-tierra [2].

La tasa de fallas por flameo inverso (BFR por sus siglas en inglés) se expresa en unidades de fallas por 100 kilómetro por año, y se producen cuando un rayo impacta la torre o el cable de guarda de una línea de transmisión, provocando una diferencia de potencial lo suficientemente elevada como para exceder el nivel de aislamiento de la línea [3]. Para lograr que el BFR sea bajo se hace necesario disponer de un buen sistema de puesta a tierra que garantice que la corriente del rayo sea dispersada en el suelo de forma efectiva [4].

Los sistemas de puesta a tierra (SPT) ofrecen una variedad de formas en su diseño, los cuales dependen del terreno y de los electrodos a usarse, siendo algunos más eficientes dependiendo de la situación [5]-[7]. En algunas estructuras es común encontrar electrodos verticales y contrapesos en los sistemas de puesta a tierra [4],[8], los cuales tienen construcción y costos diferentes que son factores importantes para tener en cuenta en el diseño.

Para analizar el efecto de los rayos en las estructuras, se tienen en cuenta las ondas viajeras a lo largo del sistema eléctrico y las ondas reflejadas que junto con la cresta de la onda del rayo son determinantes en los picos de tensión vistos en los aisladores [10],[11]. La onda reflejada depende de la velocidad con que esta se desplace, que por lo general está entre el $85 \%$ y el $90 \%$ de la velocidad de la luz [4],[8],[9], y del tiempo de viaje de las reflexiones que provienen de la puesta a tierra de la propia torre en la que impacta el rayo y de las tierras de torres adyacentes [12].

La tasa de falla por flameo inverso (BFR por sus siglas en inglés) se ha calculado siguiendo dos metodologías: CIGRÉ [4] e IEEE [8]. Ambas tienen diferencias en la distribución de probabilidad de la corriente de rayo, el tiempo de frente de la onda de corriente o los tiempos en los que se compara la tensión en el aislador, entre otras. En general las dos metodologías coinciden en estimar de forma analítica el efecto transitorio de las puestas a tierra de la torre impactada y las adyacentes en las tensiones que se presentan en la torre y en la cadena de aisladores. Esto lleva a simplificaciones que se pueden evitar simulando de forma directa todo el sistema de transmisión en un programa de análisis transitorio como ATP o EMTP, según se muestra en diferentes trabajos [10]-[16].

Por ejemplo, en [16] se presenta el modelado y la simulación de una línea de $132 \mathrm{kV}$ en Malasia orientada a verificar la energía absorbida por los descargadores a causa de flameo inverso y falla de aislamiento. El efecto de la resistencia de puesta a tierra en la tasa de fallas por flameo inverso se ha desarrollado en diferentes trabajos [4],[17]-[20].

Hilleman [4] presenta la variación del BFR en función de la resistencia de la puesta a tierra y de la longitud de los contrapesos para líneas de alta tensión y de distribución, calculada mediante la metodología CIGRÉ.

En [19] se estudia el efecto que tiene la adición de conductores verticales a los contrapesos de una línea de $161 \mathrm{kV}$, haciendo un estudio paramétrico detallado en ATP para conductividades entre 500 y $3000 \Omega$.m. Se encuentra como resultado una mejora significativa de la tasa de fallas, debido al incremento de la corriente crítica, aun cuando su efecto no es significativo a frecuencia industrial.

Visacro et al [20] revisa los requerimientos de contrapesos largos en el cálculo del BFR, concluyendo que la longitud efectiva del contrapeso es mayor que la de electrodos horizontales y de cuatro estrellas. Montaña [21] da importantes recomendaciones para los sistemas de puestas a tierra de protección contra rayos, entre las que se pueden destacar que los contrapesos son efectivos para longitudes cercanas a los $70 \mathrm{~m}$ para terrenos de alta resistividad y de $40 \mathrm{~m}$ para baja resistividad, que el mejor desempeño contra rayos se logra cuando se conectan varillas verticales al inicio del contrapeso y no en el extremo abierto y que el incremento de la longitud del contrapeso solo modifica la cola de la tensión, vista en el punto de inyección, y no el pico.

Shariatinasab et al [22] realiza un completo análisis de la tasa de flameos de una línea de $400 \mathrm{kV}$ modelada en EMTP. El artículo analiza el BFR para diferentes formas de electrodos: varilla, malla, contrapeso y mezcla de contrapeso y malla, usando diferentes métodos de cálculo y para conductividades entre 100 y $1000 \Omega$.m. Se concluye que la dependencia en la frecuencia de la resistencia de puesta a tierra de la torre lleva a un decremento en la la tasa de fallas, especialmente en 
terrenos de alta resistividad y densidad de descargas a tierra.

En este trabajo, se simularán los modelos de resistencia de sistemas de puesta a tierra propuestos en el estándar IEEE 1243-1997: tierra suplementaria con varillas y con contrapesos en el desempeño de una línea de transmisión de $230 \mathrm{kV}$ ante rayos, para diferentes valores de conductividad y haciendo uso del software ATPDraw, lo cual permitirá obtener resultados más realistas en contraste con los métodos tradicionales (CIGRÉ e IEEE) [4], [8]. Se calculará de forma detallada el efecto del número de electrodos, radio y enterramiento en los porcentajes de reducción de la tasa de fallas de la línea.

Este documento está dividido en cuatro secciones principales: la sección 2 se centra en la metodología donde se presentan los modelos empleados del sistema eléctrico en ATPDraw para simular el BFR en cada caso; en la sección 3 se exponen los resultados obtenidos del BFR para cada SPT; finalmente en la sección 4 se dan las conclusiones del estudio realizado.

\section{Metodología}

\subsection{Modelado en ATPDraw}

El sistema por analizar está compuesto por tres torres y una línea larga en sus extremos (Figura 1), cada una de suspensión auto soportada de circuito doble triangular vertical, con doble cable de guarda, una altura total de $48,6 \mathrm{~m}$, un ancho de 13,6 m y una tensión de servicio de $230 \mathrm{kV}$ como se muestra en la Figura 2. La torre central será el punto de análisis del comportamiento ante una descarga atmosférica. Las torres adyacentes están separadas $500 \mathrm{~m}$ y se diseñan de forma simplificada como una sola impedancia característica que representa la estructura total de la torre. En estas se reflejan las ondas viajeras que recorren el cable de guarda y las estructuras cercanas, las cuales son de vital importancia en la tensión vista en los aisladores [4]. Las dos torres en los extremos se ubican a $2.5 \mathrm{~km}$ del sistema a analizar y las reflexiones de las ondas en estas torres llegan en un rango de tiempo que no influye en la tensión vista en los aisladores de la torre impactada.

\subsubsection{Modelado de la estructura}

La estructura de $230 \mathrm{kV}$ mostrada en la Figura 2 se modela en ATPDraw con impedancias características para cada sección de la estructura: el cuerpo y los brazos o crucetas en donde se encuentran los aisladores, los cuales son el punto donde se presentará el flameo. Para esto se utiliza la herramienta "LINE_ZT" con sus respectivas distancias y con una velocidad de propagación de la onda del $90 \%$ de la velocidad de la luz [4][14]. Esto se puede ver en la Figura 3. Para obtener sus valores, se utiliza el modelo Sargent-Darveniza para estructuras cónicas (ecuación. 1 [3],[23]):

$$
Z_{T}=30 * \ln \left(\frac{2\left(h^{2}+r^{2}\right)}{r^{2}}\right)
$$

Donde,

$h$ es la altura de la torre o del cono en $[m]$;

$r$ es el radio de base de la torre o del cono en $[\mathrm{m}]$.

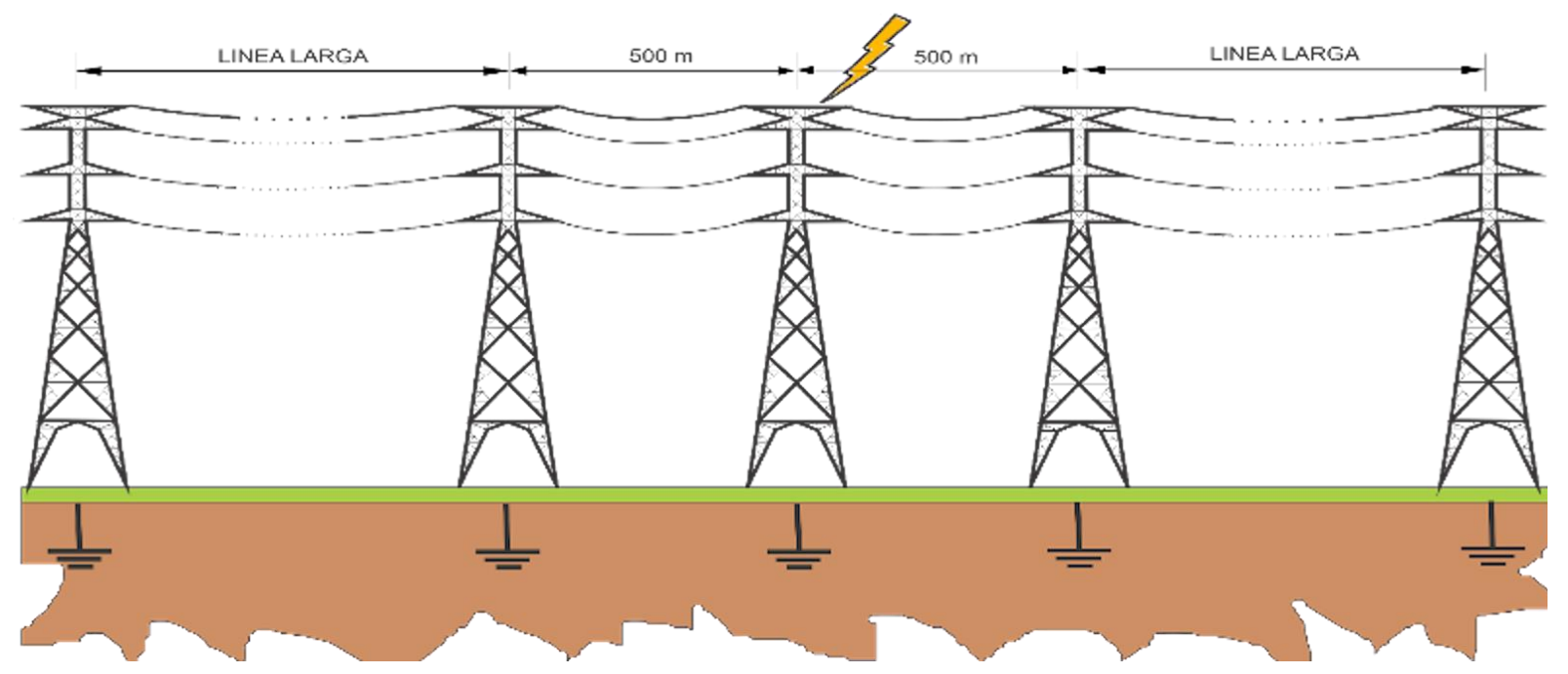

Figura 1. Representación de un tramo de la línea de transmisión de 230 kV. Fuente: elaboración propia. 
$\mathrm{Z}_{\mathrm{T} 1}$ representa la impedancia característica de la parte central vertical de la estructura compuesta por las patas, el tronco la estructura inferior y la estructura superior; $\mathrm{Z}_{\mathrm{T} 2}$ representa la impedancia característica de cada uno de los brazos de la torre (Figura 2); y $\mathrm{R}_{\mathrm{T}}$ representa la resistencia de puesta a tierra. Para el modelo se obtiene que la impedancia del tronco de la torre es $\mathrm{Z}_{\mathrm{T} 1}=139.09 \Omega$ y la impedancia de los brazos o crucetas de la torre es $\mathrm{Z}_{\mathrm{T} 2}=97.17 \Omega$ de acuerdo con la ecuación. 1 .

Para modelar en ATPDraw el tramo descrito del sistema de transmisión de $230 \mathrm{kV}$ (Figura 1), se tienen en cuenta la estructura, los aisladores, la línea, los sistemas de puesta tierra y el rayo. El circuito general simulado se presenta en la Figura 4.

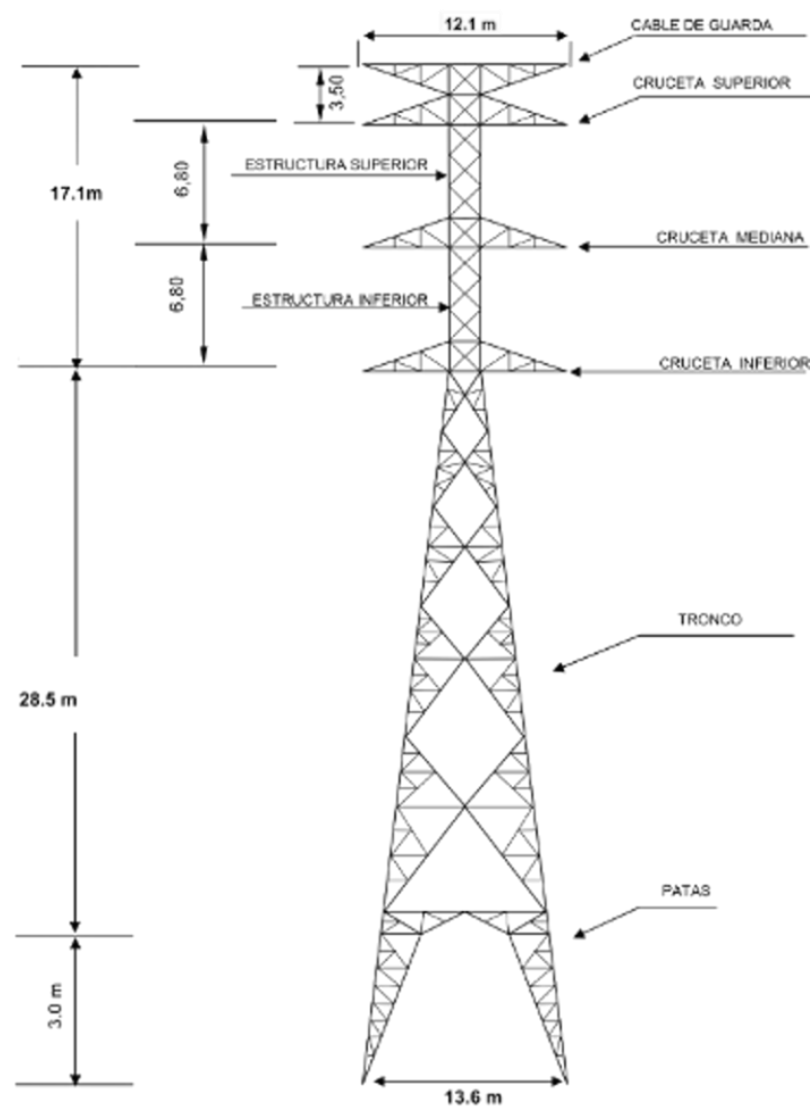

Figura 2. Torre de suspensión auto soportada de circuito doble triangular vertical con doble cable de guarda 230 $\mathrm{kV}$. Fuente: elaboración propia.

\subsubsection{Modelado de los aisladores}

Un aislador tiene un comportamiento no lineal ante impulsos atmosféricos, por tanto, la tensión soportada por una cadena de aisladores se representa en una curva dependiente del tiempo y de la longitud de la cadena compuesta por discos (ecuación 2) [8]. Esta curva propuesta por el CIGRÉ [15] es un indicador de flameo si es excedida por la tensión vista en los aisladores al caer un rayo. En ATPDraw este modelo de aislador se integró en el software mediante la herramienta "model" para diseñar la curva de tensión del aislador y "switch" para que sea el que actúe como interruptor ante un flameo utilizando la curva generada por el "model" [18]:

$$
V_{\text {Impulseflashover }}(t)=\left(400+\frac{710}{t^{0.75}}\right) W
$$

Donde,

$V$ es la tensión necesaria para que se presente flameo en $[\mathrm{kV}]$;

$t$ es el tiempo para el flameo en $[\mu \mathrm{s}]$ para $(0.5 \mu \mathrm{s}-16 \mu \mathrm{s})$; $W$ es la longitud de los aisladores en [m].

Para modelar la curva en ATPDraw, se utilizó una longitud de la cadena de aisladores de 2 metros, de acuerdo a lo establecido en IEEE 1243-1997 para 230 $k V$, obteniendo así la Figura 5.

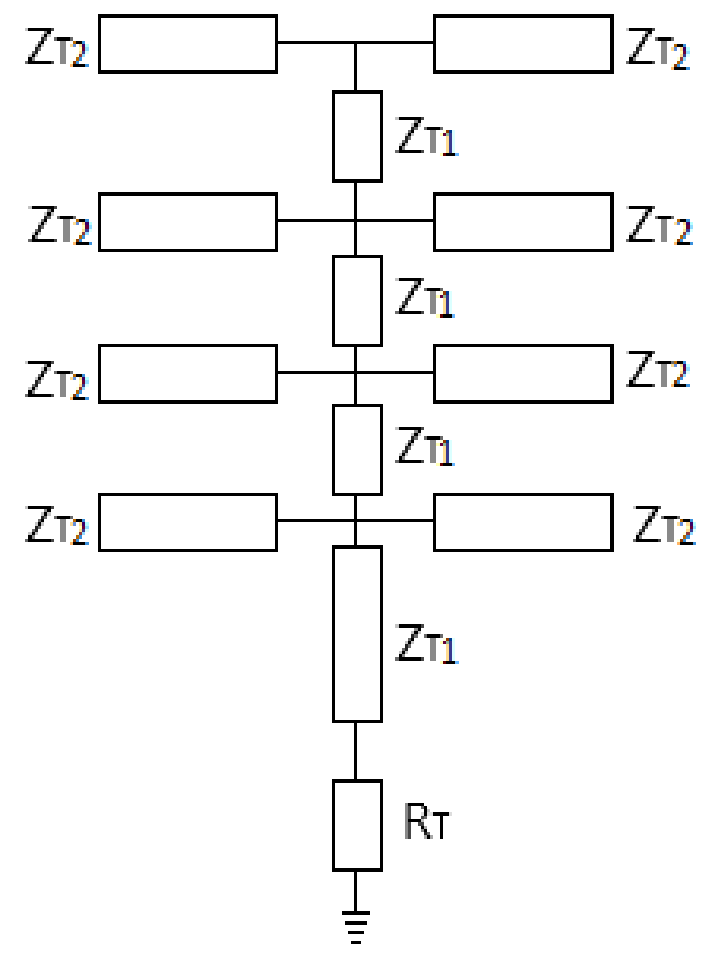

Figura 3. Modelo de impedancias de la torre doble circuito para ATPDraw. Fuente: elaboración propia. 


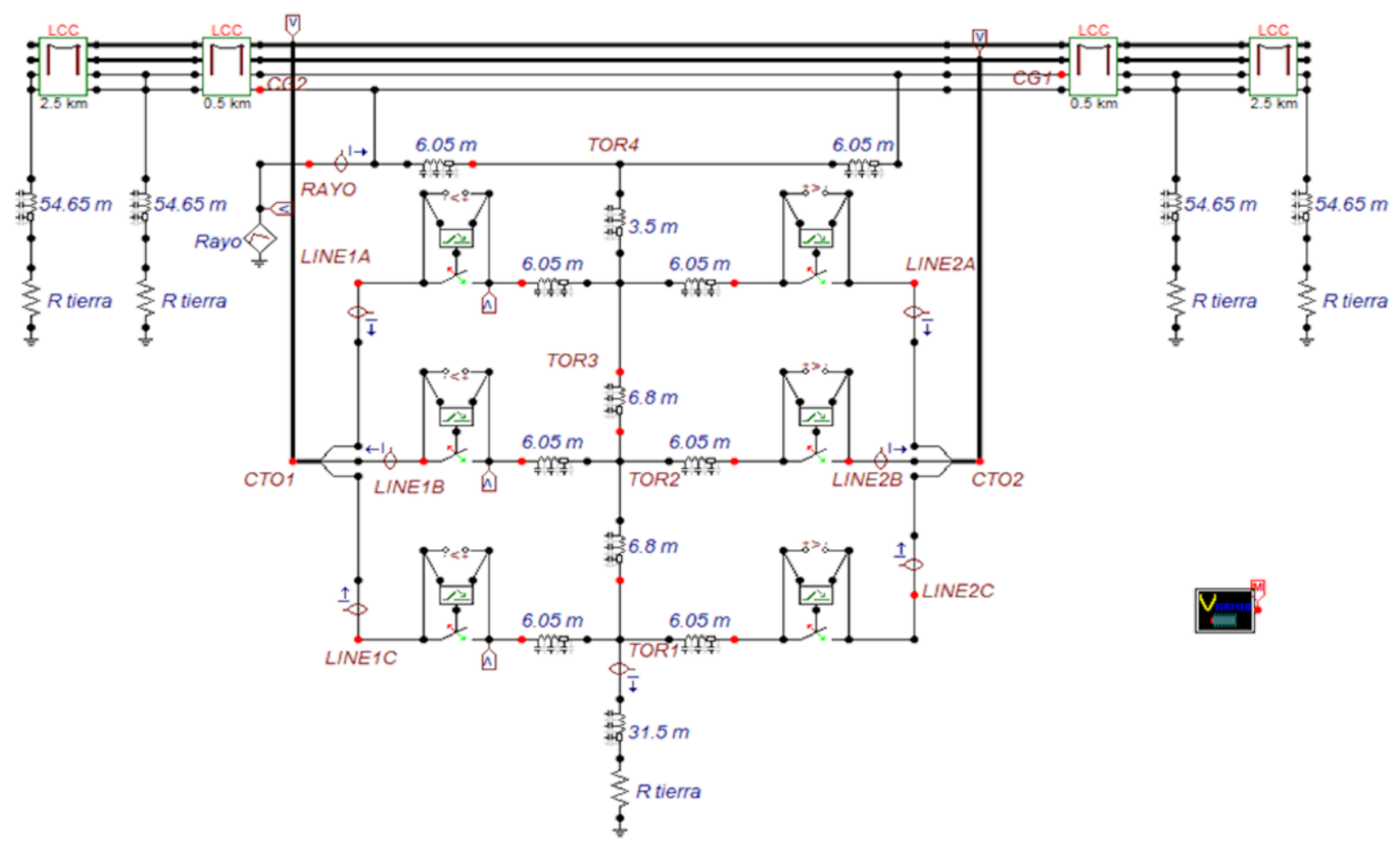

Figura 4. Modelo para la obtención de corriente crítica por flameo inverso. Fuente: elaboración propia.

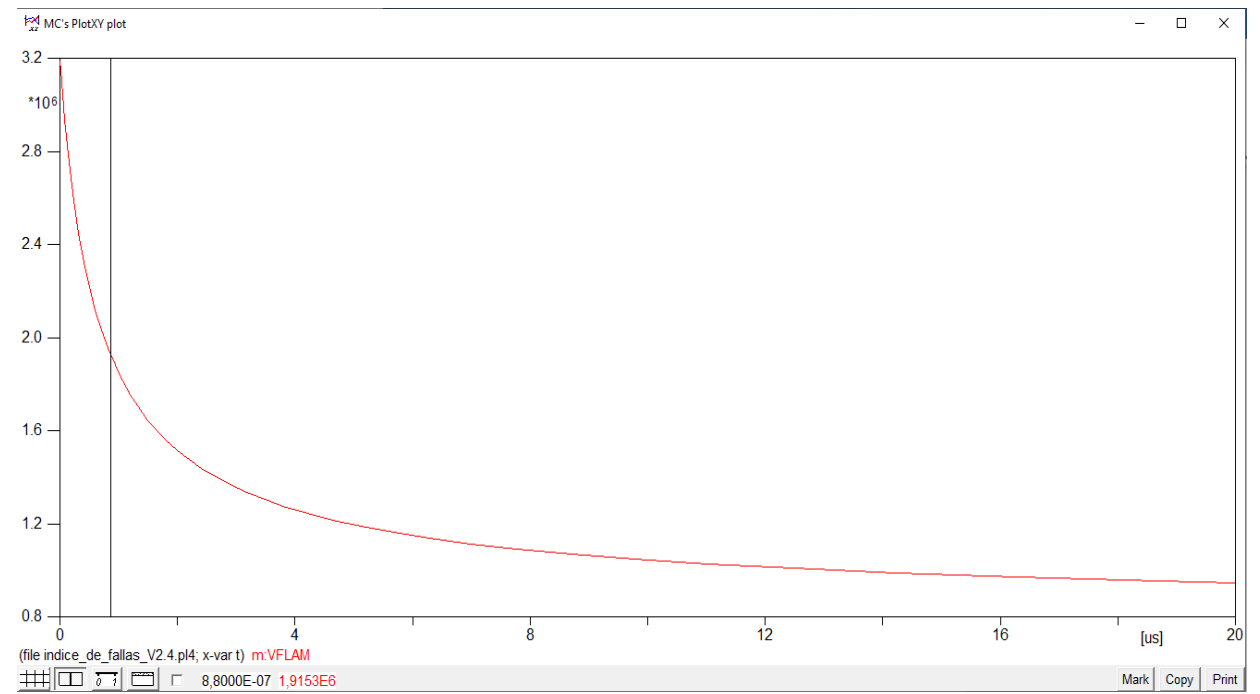

Figura 5. Tensión soportada por los aisladores en el punto crítico de la tensión con corriente de rayo de $1 \mathrm{kA}$ para una longitud de 2 metros y falla a los $0.88 \mu$ s. Fuente: elaboración propia.

\subsubsection{Modelado de la línea}

Para la línea se utiliza un cable de fase tipo FLINT AAAC con resistencia de $0.089 \Omega / \mathrm{km}$ y $2.52 \mathrm{~cm}$ de diámetro, con un cable de guarda tipo Alumoweld 7 No. 9 AWG con resistencia de $1.844 \Omega / \mathrm{km}$ y $8.71 \mathrm{~mm}$ de diámetro. En ATPDraw se utiliza la herramienta "LCC", en la que se selecciona el modelo Bergeron [24] para parámetros distribuidos de la línea, teniendo en cuenta entre otros, la resistividad del terreno que varía dependiendo del tipo de suelo (Tabla 1), y la frecuencia a la cual viaja la onda, asumida como $100 \mathrm{kHz}$; se asume un vano de $500 \mathrm{~m}$ para las torres adyacentes que producen las reflexiones más importantes (se asumió esa 
longitud como un valor promedio válido para diferentes tipos de terrenos: planos y no planos), y de $2.5 \mathrm{~km}$ para representar una línea larga, en la cual las reflexiones sobrepasan el tiempo de los $20 \mu \mathrm{s}$, a un $90 \%$ de la velocidad de la luz [4], [17], las cuales no entran a alterar los datos de la simulación.

\subsubsection{Modelado de los sistemas de puesta a tierra}

En el estándar IEEE 1243-1997 [8] se describen matemáticamente los modelos de los sistemas de puesta a tierra que en el software ATPDraw se representarán por valores resistivos.

- Tierra suplementaria con varillas: Son varillas de cobre o aleaciones de este que hacen contacto con el terreno, dispuestas de forma vertical (Figura 6) cuyos parámetros son resistividad, radio, profundidad de enterramiento y número de electrodos que conforman el sistema de puesta a tierra (ecuación 3) [8].

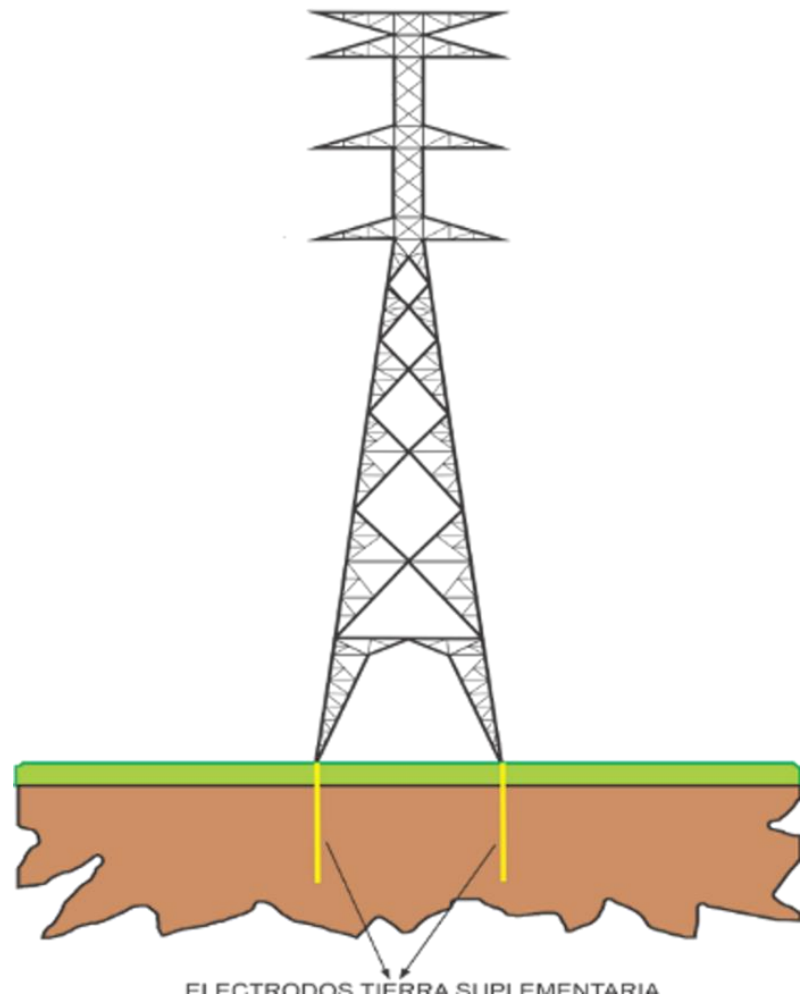

Figura 6. Sistema de puesta a tierra con electrodos verticales de tierra suplementaria. Fuente: elaboración propia.

$$
R=\frac{\rho}{2 \pi} \ln \left(\frac{2 s}{r}\right)
$$

Donde,

$R$ es la resistencia $[\Omega]$;

$\rho$ es la resistividad del suelo $[\Omega . \mathrm{m}]$;

$s$ es la longitud de la varilla en contacto con la tierra

$[\mathrm{m}]$

$r$ es el radio de la varilla [m].

De acuerdo a la normativa colombiana la longitud del electrodo mínima es de $2.4 \mathrm{~m}$ [25], sin embargo, en el mercado existen varillas de otras medidas que se pueden unir hasta lograr la longitud deseada del electrodo, no obstante, para poder tener una cantidad de datos considerables y apreciables, se utilizarán varillas de cobre de $1.8 \mathrm{~m}$ de longitud hasta llegar a una profundidad de enterramiento de $10.8 \mathrm{~m}$, siendo la unión de 6 varillas en serie utilizando uniones mecánicas y soldadura exotérmica hasta completar aproximadamente $10 \mathrm{~m}$ de longitud que es el valor máximo, basado en recomendaciones prácticas [5].

Es de aclarar que no es recomendable el uso de electrodos verticales de más de 6 metros de longitud para el comportamiento transitorio de puestas a tierra, debido a que se incrementa el comportamiento inductivo a altas frecuencias lo cual aumenta el valor de cresta de la señal de tensión, sin embargo, el efecto no se verá en este artículo, dado que la resistencia se tratará como un elemento concentrado. Para 3 radios de varilla distintos aceptados en la normativa colombiana que son $1 / 2$ ", $9 / 16$ " y $5 / 8$ " de diámetro [26]; y con 2 , 4 y 8 varillas dispuestas en forma paralela, cuya resistencia se calcula de acuerdo al equivalente de resistencias en paralelo, como una aproximación que se cumple sobre todo cuando la distancia entre electrodos es más del doble del enterramiento (Figura 8 de [8]) [4][5]. Como ejemplo, en el caso de 2 electrodos, la resistencia equivalente es la mitad de la resistencia obtenida con un solo electrodo, de acuerdo a la ley de Ohm y las leyes de Kirchoff.

Para un SPT con una resistividad de $100 \Omega$.m, dos electrodos de longitud de $7.2 \mathrm{~m}$ y $5 / 8$ " de diámetro, la resistencia (ecuación 3) es $8.29 \Omega$ aproximadamente.

- Tierra suplementaria con contrapesos: Está compuesto por cables de cobre o ACSR desnudos tendidos de forma horizontal y paralela a los conductores de fase a lo largo de la línea de transmisión (Figura 7), cuyos parámetros son la resistividad, radio, profundidad de enterramiento, longitud y número de cables que conforman el sistema de puesta a tierra deñ contrapeso (ecuación 4) [8]. 


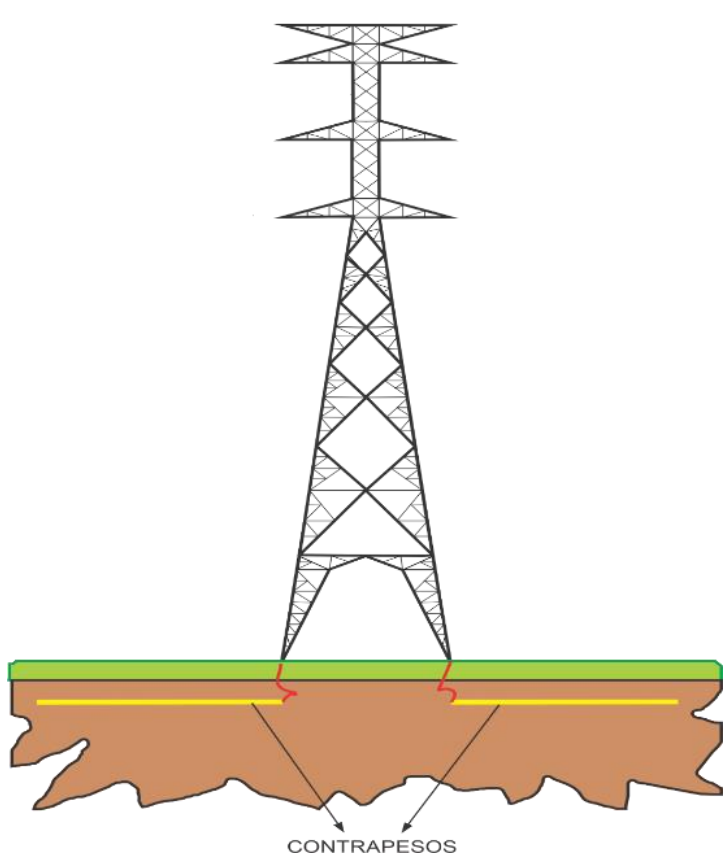

Figura 2. Sistema de puesta a tierra compuesta por contrapesos. Fuente: elaboración propia.

$$
R=\frac{\rho}{\pi s}\left(\ln \left(\frac{2 s}{\sqrt{4 r d}}\right)-1\right)
$$

Donde,

$R$ es la resistencia $[\Omega]$;

$r$ es el radio del cable $[\mathrm{m}]$

$d$ es la profundidad de enterramiento [m];

$s$ es la longitud del contrapeso [m].

$\rho$ es la resistividad del suelo [ $\Omega . \mathrm{m}]$;

Para el modelo de contrapeso se utilizarán 1 y 2 cables de cobre como contrapesos, con profundidades de enterramiento de 0.5 y $1.5 \mathrm{~m}$; calibres de $2 / 0$ y $4 / 0$ AWG; y longitudes de 10 hasta $100 \mathrm{~m}$, de acuerdo con lo dispuesto en la IEEE 1243-1997.

La resistencia de éste SPT teniendo una resistividad de

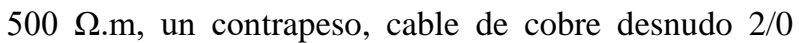
AWG, con una longitud de 70 metros y enterrado a una profundidad de $0.5 \mathrm{~m}$ es de $14.13 \Omega$ (Ec. 4); en caso de que sean dos contrapesos, despreciando los efectos de ionización del suelo y de mutua resistencia entre los cables, tenemos aproximadamente una resistencia de 7.1 $\Omega$.

El suelo tiene diferentes características que afectan la capacidad de conducir electricidad, como la humedad, composición química, compactación del material, concentración de sales, temperatura, entre otros; por tanto, los terrenos no tienen una resistividad homogénea ya que cada punto del terreno tiene características diferentes, sin embargo, se pueden clasificar entre un rango de valores que permiten determinar el tipo de terreno como se muestra en la (Tabla 1), y para determinar estos valores se usa un equipo de medición llamado telurómetro.

Tabla 1. Resistividad por clase de suelo

\begin{tabular}{|c|c|}
\hline Clase de suelo & Resistividad [ $\Omega . \mathrm{m}]$ \\
\hline Clase A: Blandos ácidos & Menor a 200 \\
\hline $\begin{array}{l}\text { Clase B1: Arenosos } \\
\text { semisecos }\end{array}$ & Entre 500 y 1000 \\
\hline lase B2: Arenosos secos & Entre 1000 y 2000 \\
\hline Clase C1: Rocosos & Menor a 1000 \\
\hline Clase C2: Rocosos & Mayor a 1000 \\
\hline
\end{tabular}

Fuente: tierras soporte de la seguridad eléctrica [5].

Para facilitar el análisis y poder emplear los modelos matemáticos del estándar IEEE 1243, se trabaja con una resistividad homogénea en todo el terreno, correspondiente a 4 tipos de suelos, con resistividades de $1,100,500$ y $1000 \Omega$.m.

\subsubsection{Modelado del rayo}

La fuente del rayo que se utiliza en ATPDraw es una "Slope-Ramp type 13", la cual se une a la torre para simular el impacto en la misma, teniendo una forma de onda $1.2 / 50 \mu \mathrm{s}$, teniendo en cuenta que tiempos de frente cercanos a $1 \mu$ s se han usado en otras referencias [20]. La amplitud máxima es de $1 \mathrm{kA}$, siendo este valor escalable hasta el valor necesario para superar la tensión de aislamiento del aislador. En esta simulación no se tuvo en cuenta la señal de tensión de $60 \mathrm{~Hz}$ considerando un caso simplificado en el cual la onda sinusoidal está pasando por $0 \mathrm{~V}$.

\subsection{Obtención del BFR}

La tasa de fallas por flameo inverso también conocida por siglas en inglés BFR o BFOR indica el número de fallas por impactos directos sobre la torre o el cable de guarda que producen flameos. La expresión del BFR considera que, de todos los impactos directos, los de la torre son los que pueden producir flameo en mayor medida y los estima en un $60 \%$ [3][4]:

$$
B F R=0.6 N_{L} P\left(I_{C}\right)
$$


Donde,

$N_{L}$ es la cantidad de rayos que impactan la estructura [impactos/100km/año] (ecuación 6) [4],[18]; $P\left(I_{C}\right)$ es la probabilidad de que la corriente $I_{C}$ sea excedida (ecuación 7) [3], [4].

$$
N_{L}=N_{g}\left(\frac{28 h^{0.6}+S_{g}}{10}\right)
$$

Donde,

$N_{g}$ es la densidad de descargas a tierra por unidad de superficie en un cierto lugar geográfico en [rayos $/ \mathrm{km}^{2} / \mathrm{año}$;

$h$ es la altura de la torre en [m];

$S_{g}$ es la distancia entre cables de guarda en [m].

$$
P\left(I_{C}\right)=\frac{1}{1+\left(\frac{I_{C}}{31}\right)^{2.6}}
$$

Donde,

$I_{C}$ es la corriente crítica a la cual hay falla (la tensión en la torre supera el nivel de aislamiento) [kA].

Para el ejemplo de SPT de tierra suplementaria con descrito en la seccion anterior con resistencia de 8.29 $\Omega$. Al modelar la línea en ATPDraw (Figura 3), se procede a modificar los valores de los elementos de resistencia de tierra $(\mathrm{R}$ tierra=8.29 $\Omega$ ) y los valores de resistividad dentro de la herramienta "LCC" en 100 S.m, para luego simular y ver las diferentes tensiones entre cada fase y la estructura, en las cuales se busca el punto de tensión más elevado y el tiempo en el que se produce al aplicar una corriente de rayo como la descrita en la sección 2.1.5, la cual es la responsable de que se produzca flameo y corresponde a $16.4 \mathrm{kV}$ en un tiempo de $0.88 \mu$ s (Figura 8), luego, con este tiempo se busca el punto de intersección en la curva de tensión soportada por los aisladores (Figura 5) y corresponde a 1.91 MV.

La división entre estos dos valores permite obtener la corriente crítica que es de $116.6 \mathrm{kA}$, y a su vez la probabilidad de que la corriente produzca un flameo (ecuación 7), siendo esta de 3.09\%. Posteriormente, teniendo en cuenta una densidad de rayos en la zona de 1 rayo/ $/ \mathrm{km}^{2} / \mathrm{año}$, se obtiene la cantidad de rayos que impactan la estructura (ecuación 6), que es 29.99 rayos $/ \mathrm{km}^{2} / a$ ño, y con estos valores en la (ecuación 5), se obtiene el BFR de 0.6 aproximadamente.

Para determinar el valor del BFR se usa la ecuación 5, en la cual se puede observar que el resultado está relacionado con los cambios de resistencia del SPT, pues el valor de $P\left(I_{C}\right)$ depende de la corriente crítica de cada evento, y entre menor sea el valor de resistencia a tierra mayor corriente crítica va a ser necesaria para provocar la falla, por lo que entre más alta sea dicha corriente menor va a ser la probabilidad de que ésta ocurra, por tanto, el valor del BFR será bajo, y un valor bajo es lo que se busca pues indica pocas fallas en el transcurso de un año.

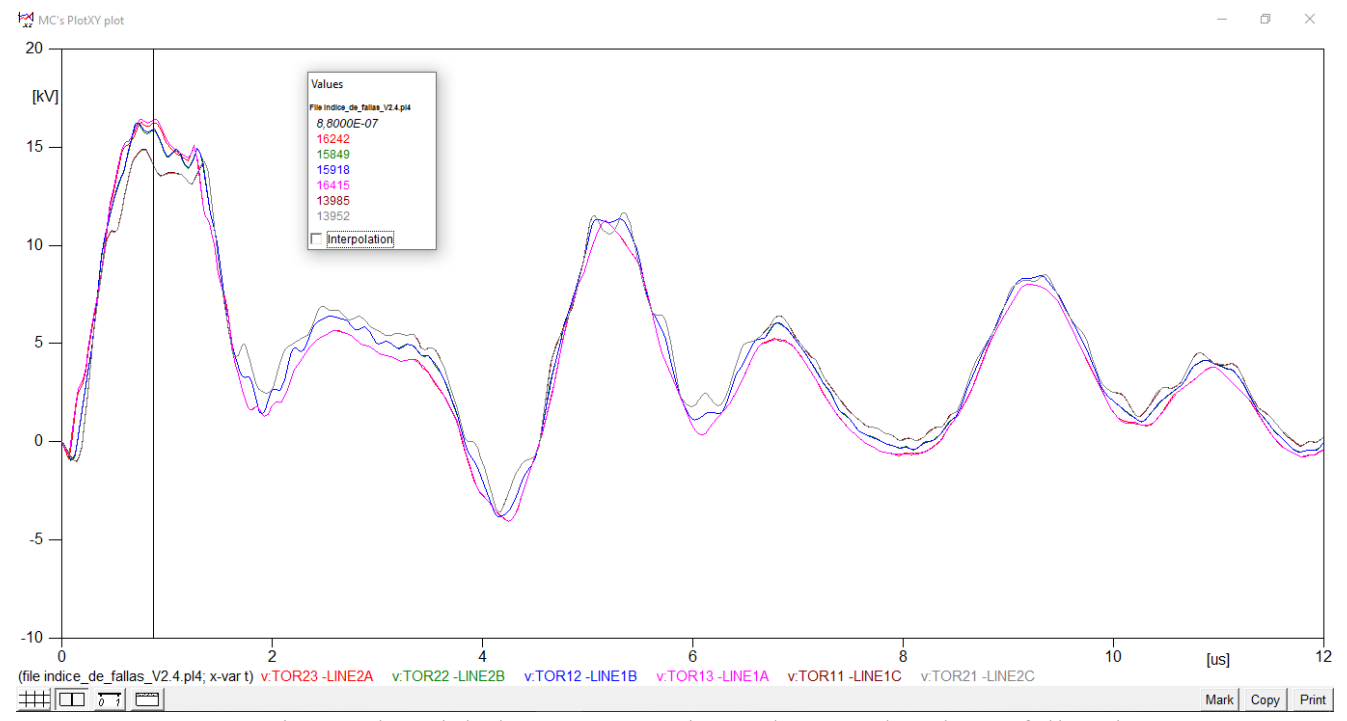

Figura 8. Tensión en los aisladores con corriente de rayo de $1 \mathrm{kA}$ y falla a los $0.88 \mu \mathrm{s}$.

Fuente: elaboración propia. 


\section{Resultados}

En esta sección se analiza el BFR para configuraciones de SPT de tierra complementaria con varillas y contrapesos para resistividades del suelo constantes de 1 , 100,500 y $1000 \Omega . m$. En cada caso, se utilizaron longitudes y diámetros de electrodos de acuerdo con lo establecido en la norma NTC 2206, el RETIE y la IEEE 1243-1997. La normativa colombiana especifica $20 \Omega$ como máxima resistencia permitida para estructuras [25], sin embargo, para efectos de estudio y análisis, se utilizaron datos de resistencia mayores a la norma.

\subsection{Tierra suplementaria con varillas}

En las Figuras 9, 10, 11 y 12 se muestra el BFR en función de las longitudes de los electrodos (Eltd), variando a) el radio entre $1 / 2$ ", $9 / 16$ " y $5 / 8$ " y b) la cantidad de los electrodos entre 2, 4 y 8 para los distintos escenarios de resistividades propuestos. También se muestra la resistencia asociada a cada punto en la curva de mayor valor de BFR. Así, cada figura indica el desempeño de las configuraciones del SPT de tierra suplementaria con varillas ante descargas atmosféricas.

En la Figura 9, para una resistividad de $1 \Omega . \mathrm{m}$, se puede ver que el valor del BFR es el mismo, dado que la resistencia de puesta a tierra varía de forma pequeña a medida que aumenta la longitud, el radio y la cantidad de electrodos. Para una resistividad de $100 \Omega$.m (Figura 10), se aprecia que el valor más alto del BFR es de 2.3 fallas $/ 100 \mathrm{~km} / \mathrm{año}$, correspondiente a 2 electrodos y $1.8 \mathrm{~m}$ de longitud; al utilizar 4 electrodos el BFR se reduce en un $61 \%$ y con 8 electrodos en un $83 \%$. Para una longitud de $9 \mathrm{~m}$ o superior, se logra una máxima reducción del $83 \%$ utilizando 2,4 u 8 electrodos, observándose que a partir de este punto no existe variación apreciable del BFR. En la Figura 11, se presenta el resultado para una resistividad de $500 \Omega$.m. En este caso el máximo valor del BFR es de 10.8 fallas $/ 100 \mathrm{~km} /$ año. Para una longitud de $5.4 \mathrm{~m}$ y utilizando 2 electrodos el BFR se reduce en un $56 \%$, con 4 electrodos en un $81 \%$ y con 8 electrodos en un $93 \%$.

Adicionalmente, se evidencia practicamente indepedencia en el radio de los electrodos. Finalmente para una resisitividad de 1000 S.m (Figura 12), variando el radio y la cantidad de los electrodos se aprecia que el valor más alto del BFR es de 14.7 fallas/100km/año. Para una longitud de $7.2 \mathrm{~m}$ y utilizando 2 electrodos el BFR se reduce en un $51 \%$, con 4 electrodos en un $76 \%$ y con 8 electrodos en un $90 \%$. En esta resistividad se tiene un BFR alto, teniendo que utilizarse 8 electrodos y longitudes de más de $7.2 \mathrm{~m}$ para lograr una reducción de hasta un $94 \%$ y que esté acorde a la norma de resistencia máxima permitida, consiguiéndose un aumento considerable en la cantidad de materiales necesarios para su construcción.

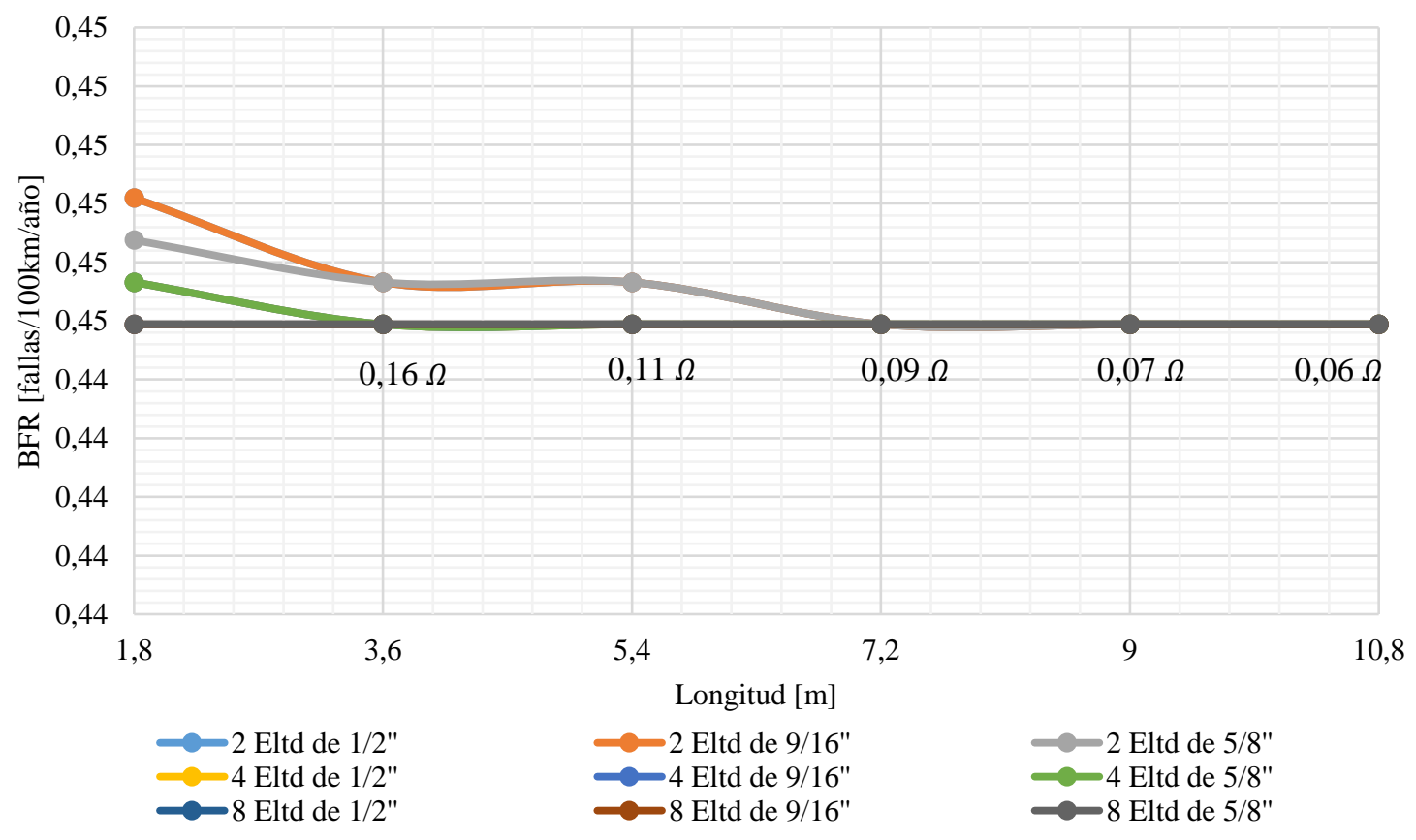

Figura 9. BFR vs Longitud de electrodos para tierra suplementaria con varillas y resistividad de $1 \Omega . \mathrm{m}$. Fuente: elaboración propia. 


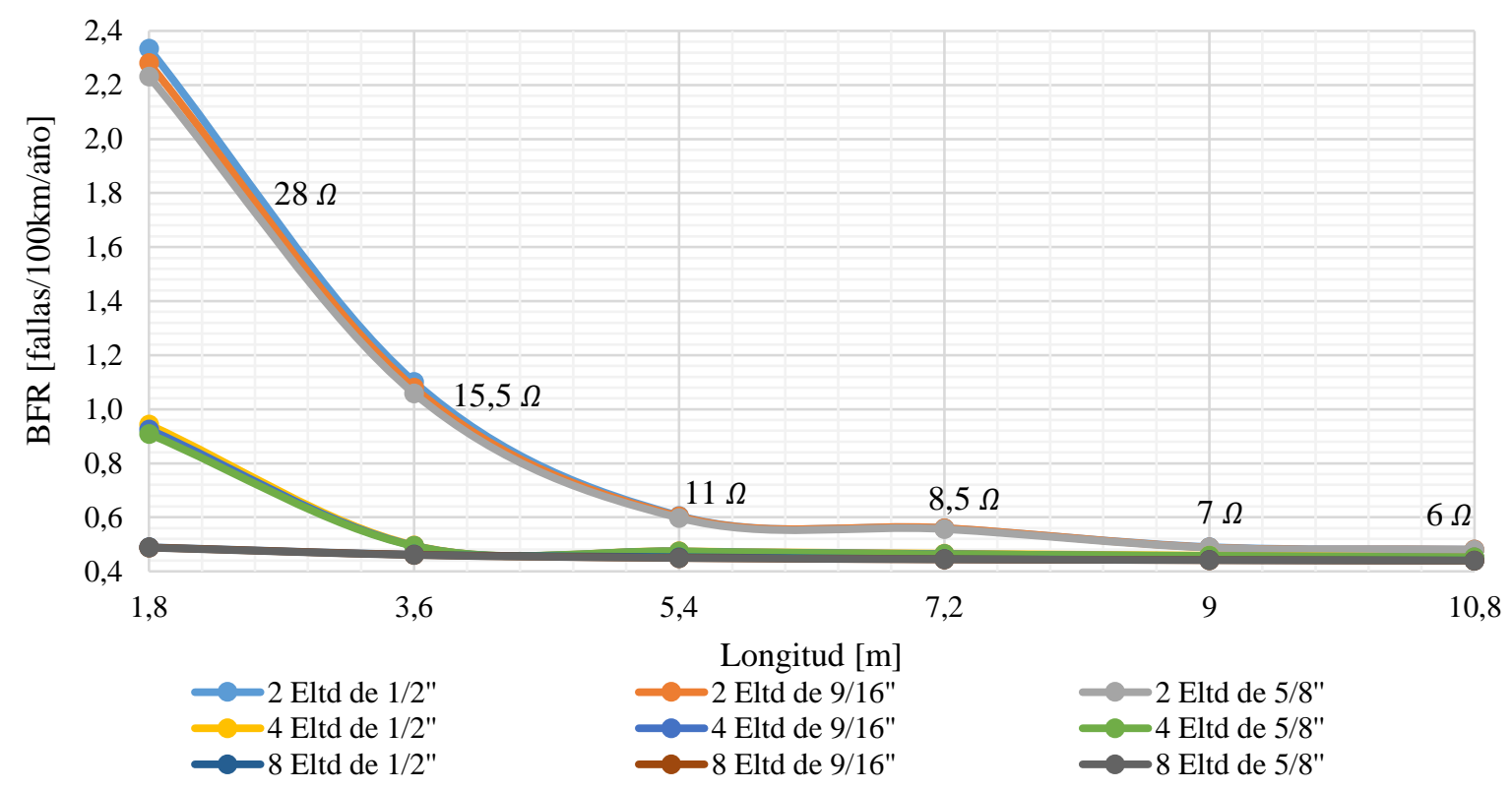

Figura 10. BFR vs Longitud de electrodos para tierra suplementaria con varillas y resistividad de $100 \Omega$.m. Fuente: elaboración propia.

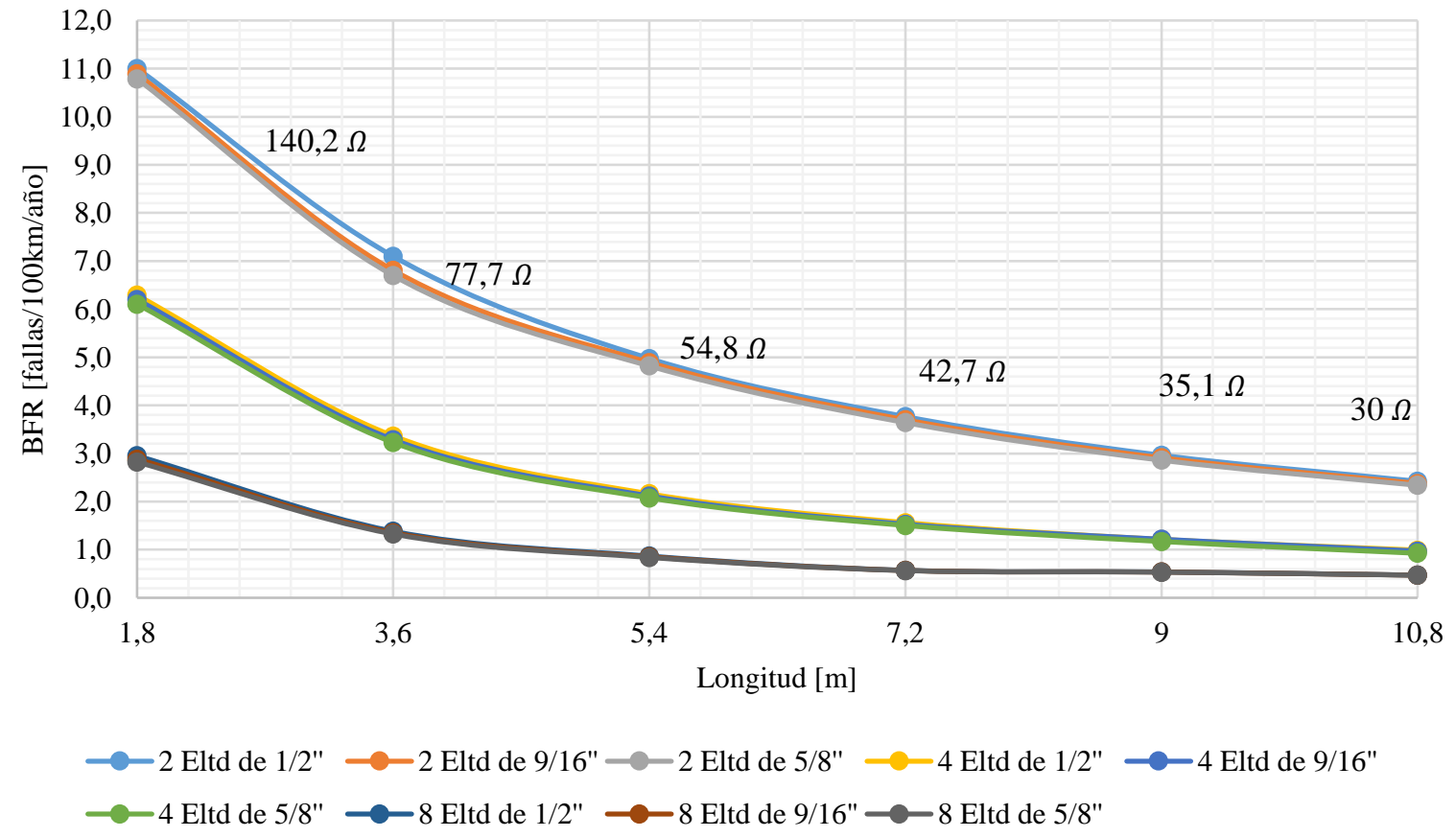

Figura 11. BFR vs Longitud de electrodos para tierra suplementaria con varillas y resistividad de $500 \Omega$.m. Fuente: elaboración propia. 
Influencia de los sistemas de puesta a tierra descritos en el estándar IEEE 1243 en la tasa de fallas por rayos de líneas de transmisión

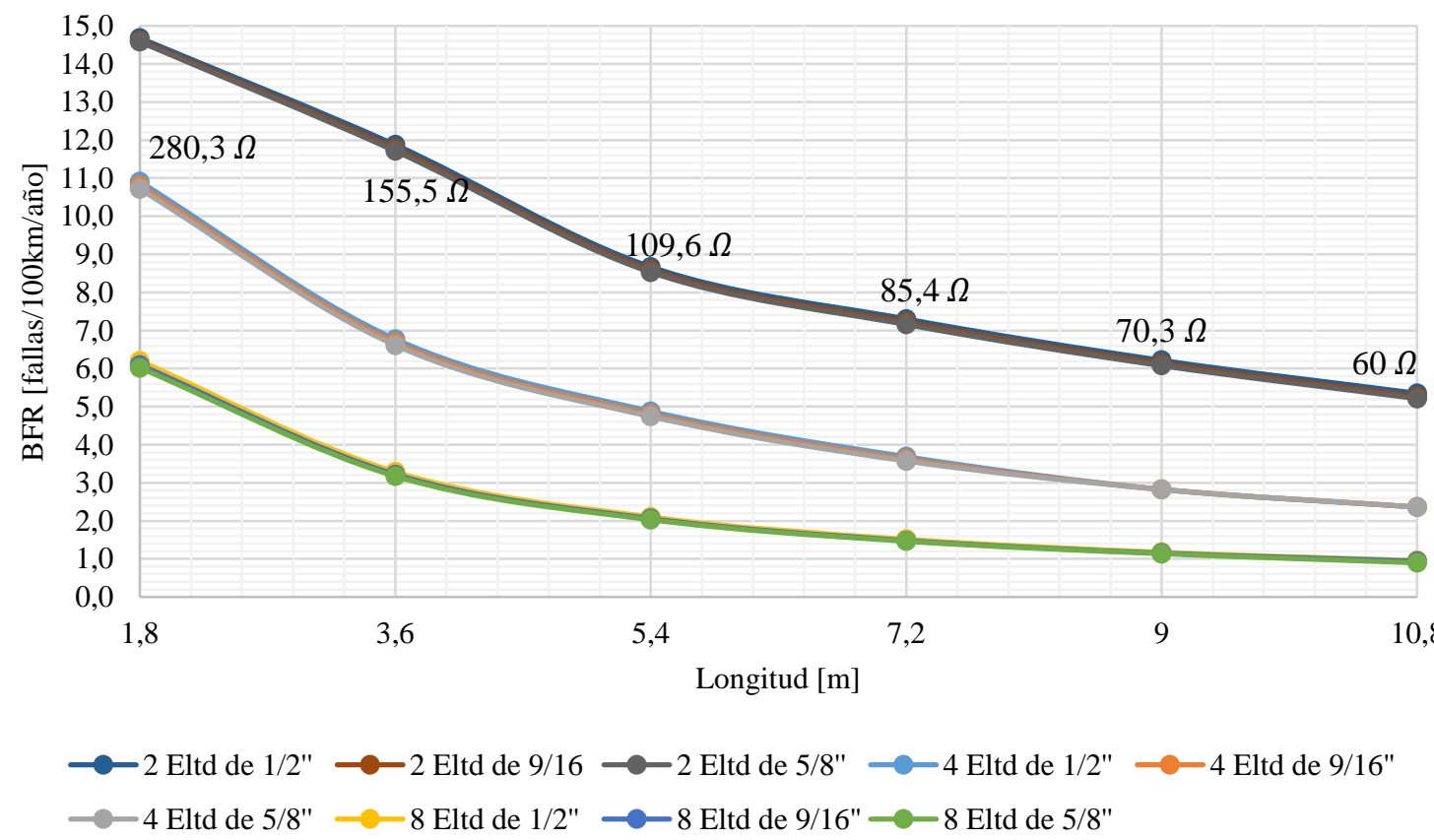

Figura 12. BFR vs Longitud de electrodos para tierra suplementaria con varillas y resistividad de $1000 \Omega$.m. Fuente: elaboración propia.

Con este tipo de SPT se evidencia que las mejores reducciones del BFR se logran con 8 electrodos, siendo los cambios más evidentes en terrenos arenosos semisecos $(500 \Omega . m)$ y terrenos rocosos $(1000 \Omega . m)$, sin embargo, a partir de cierta longitud en cada escenario no se presentan cambios significativos en la reducción del BFR, pero sí se aumenta de forma considerable la cantidad de material necesaria para construir el SPT. Al utilizar 2 electrodos, se presenta un número alto de fallas en suelos con resistividades grandes y a su vez elevadas resistencias de puesta a tierra, por lo cual, se debe tener en cuenta los valores máximos de resistencia de tierra permitidos por la normatividad nacional vigente. En general, se evidencia una reducción en el BFR cuando se aumenta el número de electrodos enterradas en paralelo, sin embargo, al cambiar el diámetro del electrodo, no se percibe un cambio apreciable en el BFR, especialmente para enterramientos o longitudes muy grandes.

\subsection{Tierra suplementaria con contrapesos}

En las Figuras 13, 14, 15 y 16 se presenta el BFR en función de las longitudes de los electrodos de contrapeso (Ctrp), variando a) el número de contrapesos entre 1 ó 2 b) El calibre de los conductores entre 1/0, 2/0 y 4/0 y c) enterramientos de 0.5 o $1.5 \mathrm{~m}$. Se consideraron los mismos valores de resistividad que en la sección 3.1. También se presenta la resistencia asociada a cada punto en la curva de mayor valor de BFR. Las gráficas están creadas para contrapesos de 10 hasta $100 \mathrm{~m}$ de longitud de acuerdo con valores prácticos [4].

En la Figura 13, se describe el cambio del BFR a medida que se aumenta la longitud del cable del contrapeso con resistividad de $1 \Omega . m$. En este escenario no hay variación significativa del BFR, debido al pequeño cambio en la resistencia en función de la longitud, el calibre o el enterramiento. Para una resistividad de 100 $\Omega . m$, mostrada en la Figura 14, En este escenario se aprecia que el valor más alto del BFR es de 0.9 fallas/100km/año, correspondiente a 1 contrapeso de 10 $\mathrm{m}$ de longitud y enterramiento de $0.5 \mathrm{~m}$; al utilizar 1 contrapeso con enterramiento de $1.5 \mathrm{~m}$ el BFR se reduce en un 32\%; al utilizar 2 contrapesos con enterramiento de $0.5 \mathrm{~m}$ o $1.5 \mathrm{~m}$ el BFR se reduce en un $47 \%$. Para longitudes superiores de $30 \mathrm{~m}$ la tasa de fallas tiende a

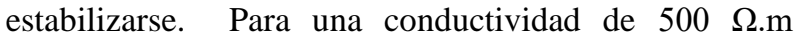
(Figura 15) se aprecia que el valor más alto del BFR es de 6.1 fallas $/ 100 \mathrm{~km} /$ año. Para $30 \mathrm{~m}$ de longitud y un contrapeso con enterramiento de $0.5 \mathrm{~m}$ el BFR se reduce en un 64\%; al utilizar 2 contrapesos con enterramiento de $1.5 \mathrm{~m}$ en un $87 \%$. Para una longitud de $80 \mathrm{~m}$ o superior el BFR se reduce en un $92 \%$ sin importar la configuración. Para 1000 S.m (Figura 16), se aprecia que el valor más alto del BFR es de 10.7 fallas/100km/año. 


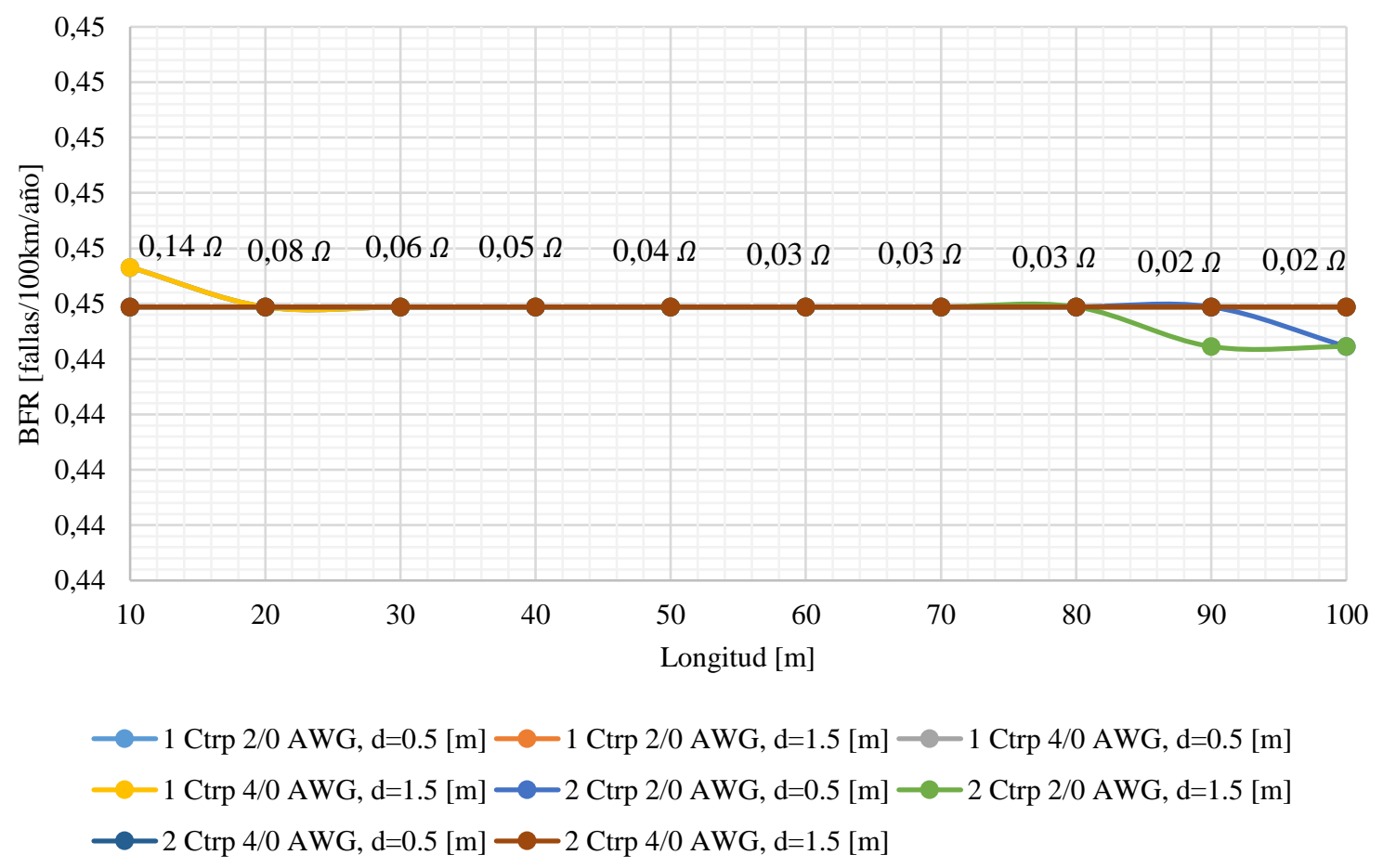

Figura 13. BFR vs Longitud de los cables de contrapesos con resistividad de $1 \Omega . \mathrm{m}$. Fuente: elaboración propia.

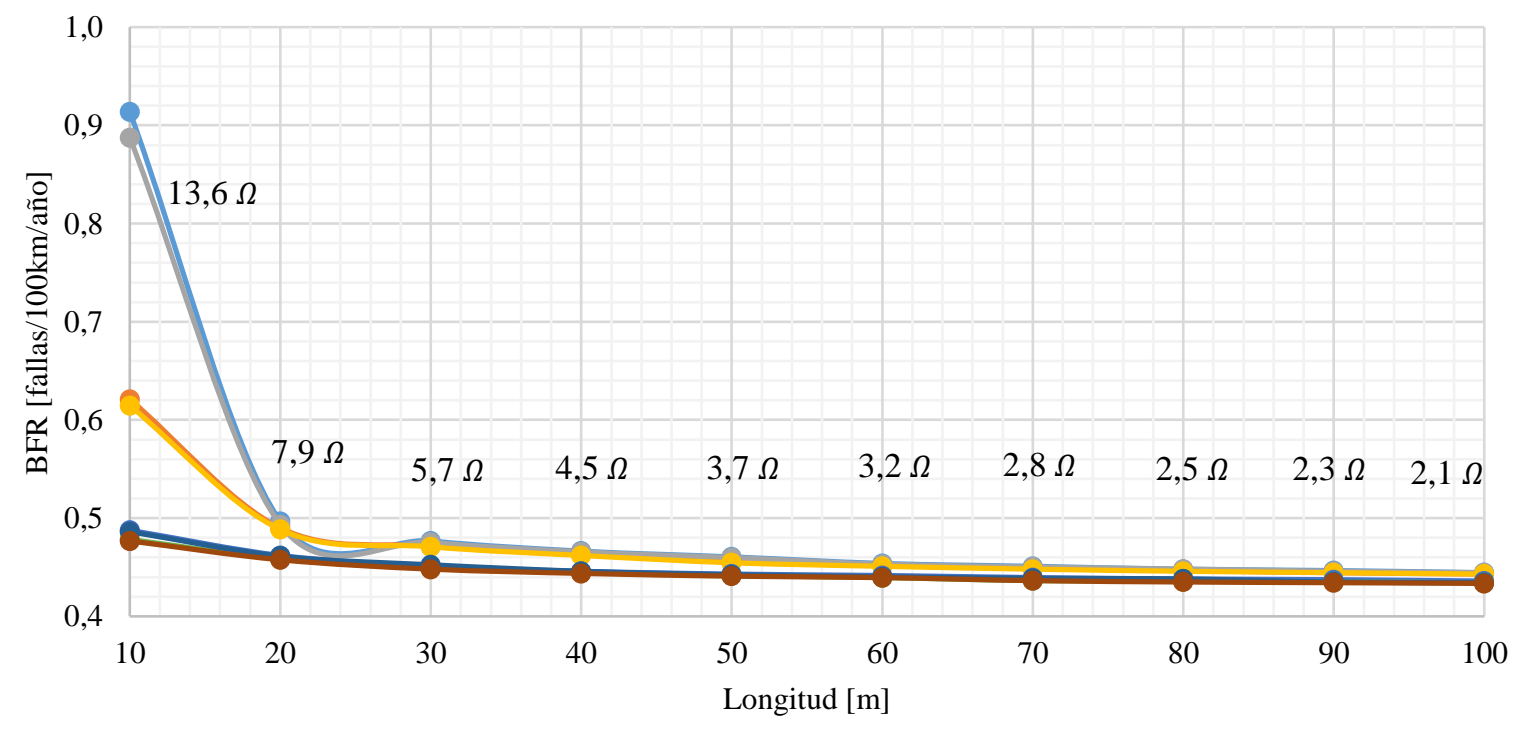

\footnotetext{
$\longrightarrow 1$ Ctrp 2/0 AWG, d=0.5 [m] $\longrightarrow 1$ Ctrp 2/0 AWG, d=1.5 [m] -1 Ctrp 4/0 AWG, d=0.5 [m]

$\longrightarrow 1$ Ctrp 4/0 AWG, $d=1.5[\mathrm{~m}] \longrightarrow 2$ Ctrp 2/0 AWG, $d=0.5[\mathrm{~m}] \multimap 2$ Ctrp 2/0 AWG, $d=1.5[\mathrm{~m}]$

$\longrightarrow 2$ Ctrp 4/0 AWG, d=0.5 [m] $\longrightarrow 2$ Ctrp 4/0 AWG, d=1.5 [m]
}

Figura 14. BFR vs Longitud de los cables de contrapesos con resistividad de $100 \Omega$.m.

Fuente: elaboración propia. 
Influencia de los sistemas de puesta a tierra descritos en el estándar IEEE 1243 en la tasa de fallas por rayos de líneas de transmisión

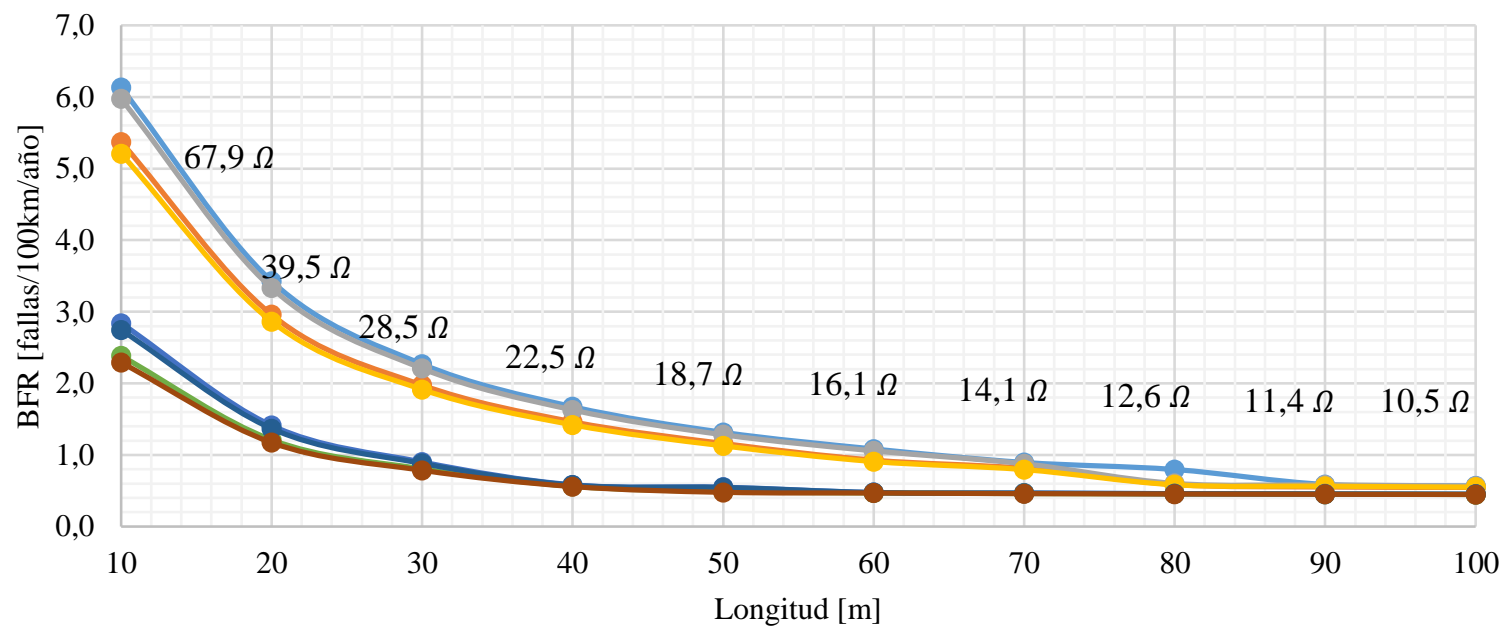

-1 Ctrp 2/0 AWG, d=0.5 [m] -1 Ctrp 2/0 AWG, d=1.5 [m] -1 Ctrp 4/0 AWG, d=0.5 [m]

-1 Ctrp 4/0 AWG, d=1.5 [m] -2 Ctrp 2/0 AWG, d=0.5 [m] 2 Ctrp 2/0 AWG, d=1.5 [m]

$\multimap 2$ Ctrp 4/0 AWG, d=0.5 [m] $\longrightarrow 2$ Ctrp 4/0 AWG, d=1.5 [m]

Figura 15. BFR vs Longitud de los cables de contrapesos con resistividad de $500 \Omega . \mathrm{m}$. Fuente: elaboración propia.

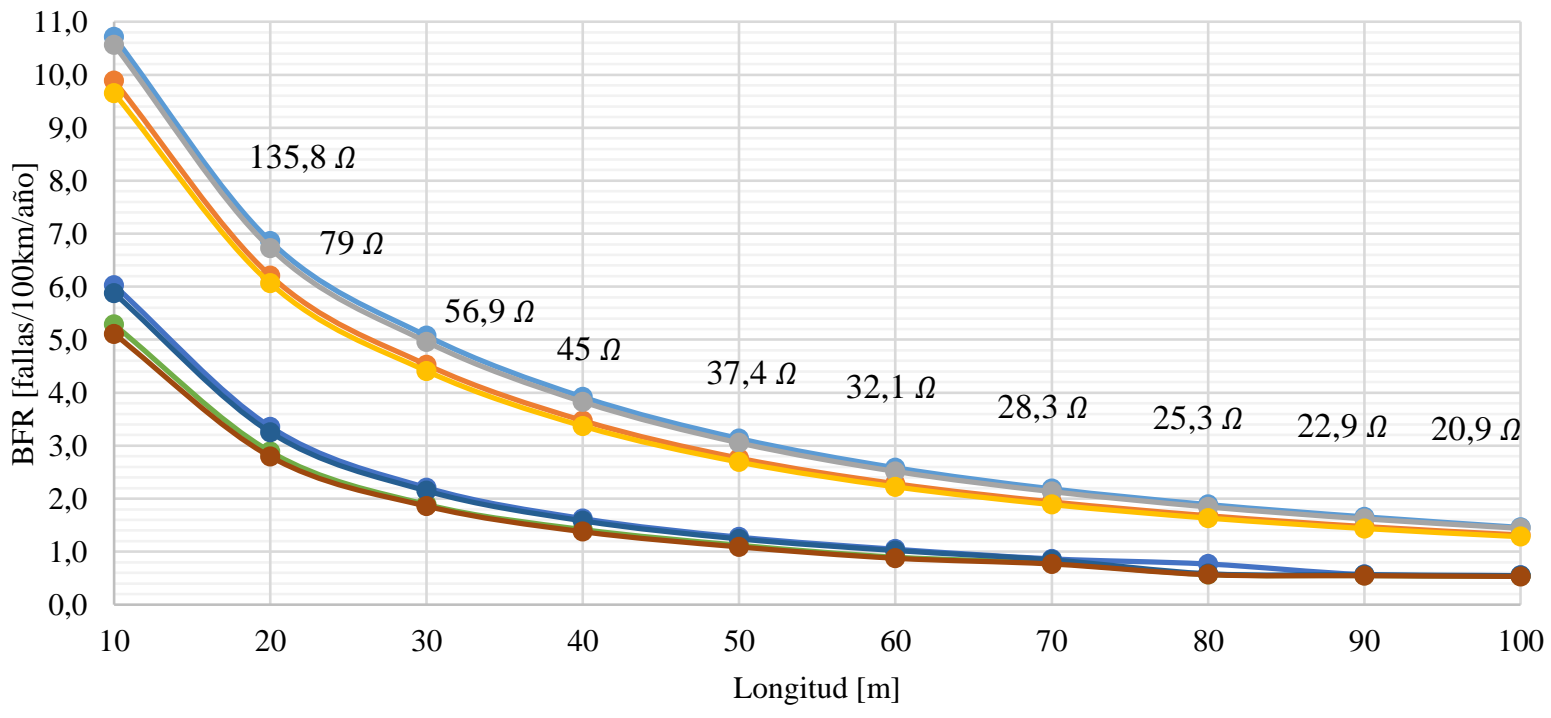

\footnotetext{
$-1 \mathrm{Ctrp} 2 / 0$ AWG, d=0.5 [m] $-1 \mathrm{Ctrp} 2 / 0$ AWG, d=1.5 [m] $-1 \mathrm{Ctrp} 4 / 0$ AWG, d=0.5 [m]

-1 Ctrp 4/0 AWG, d=1.5 [m] $\longrightarrow 2$ Ctrp 2/0 AWG, d=0.5 [m] -2 Ctrp 2/0 AWG, d=1.5 [m]

-2 Ctrp 4/0 AWG, d=0.5 [m] -2 Ctrp 4/0 AWG, d=1.5 [m]
}

Figura 16. BFR vs Longitud de los cables de contrapesos con resistividad de $1000 \Omega$.m.

Fuente: elaboración propia. 
Para $50 \mathrm{~m}$ de longitud y 1 contrapeso con enterramiento de $0.5 \mathrm{~m}$ el BFR se reduce en un $72 \%$; al utilizar 2 contrapesos con enterramiento de $1.5 \mathrm{~m}$ en un $90 \%$. En general se evidencia que un enterramiento mayor disminuye la tasa de fallas aunque no en forma significativa. La inclusión de un contrapeso adicional independiente del enterramiento, logra disminuir en mayor porcentaje la tasa de fallas.

En el SPT de contrapeso, se observa un mejor comportamiento del BFR para altas resistividades, al reducir estos valores de forma significativa, aunque se hace necesario emplear mayor cantidad de materiales y mano de obra para tender el contrapeso en grandes longitudes. En cuanto a aumentar la profundidad de enterramiento del contrapeso en $1 \mathrm{~m}$ en todos los casos estudiados, se observa que la influencia en reducir el BFR es menor al $10 \%$ y en longitudes mayores a $60 \mathrm{~m}$ la reducción es menor al 8\%. En general, se observa para este modelo que, a mayor longitud y número de contrapesos, y mayor enterramiento se producen mejoras en el BFR, sin embargo, cambiar el calibre del conductor del contrapeso, tiene un efecto casi nulo en la tasa de fallas.

\section{Conclusiones}

Para el caso de tierra suplementaria con varillas, aumentar la longitud de los electrodos tiene especial importancia para terrenos con resistividades mayores a $100 \Omega$.m. En el caso de tierra suplementaria con contrapesos, la longitud es relevante para resistividades mayores a $500 \Omega . m$.

Cuando se simularon tierras suplementarias se consiguieron reducciones del BFR de hasta el 95\%, combinando el aumento de la longitud, el número y el calibre de los electrodos. En el caso de contrapesos se logran porcentajes de disminución que van hasta el $94 \%$. Esto quiere decir que se consiguen porcentajes de disminución muy similares en ambas configuraciones.

A medida que la resistencia del sistema de puesta a tierra disminuye, el BFR también lo hace, reduciéndose también la cantidad de flameos sobre la línea, sin embargo, dependiendo de la resitividad, hay una longitud más allá de la cual, no se consiguen reducciones adicionales en la tasa de fallas.

\section{Referencias}

[1] H. Torres, "Los rayos: una visión mitológica, científica y tecnológica", Ingeniería e investigación, vol. 1, no. 24, pp. 29-34, 1991.
[2] H. Torres, "El espacio y el tiempo en los parámetros del rayo", en El rayo. Mitos, leyendas, ciencia y tecnología, $2^{a}$ ed., Bogotá, Colombia: UNIBIBLOS, 2002, pp. 123-131.

[3] CIGRE Working Group of Lightning, Guide to the procedures for estimating the lightning performance of transmission lines. CIGRE, Paris, Francia, 1991.

[4] A.R. Hileman, Insulation coordination for power systems. Boca Raton, FL, USA: CRC Press, 1999.

[5] F. Casas, Tierras: soporte de la Seguridad Eléctrica, $3^{a}$ ed. Bogotá, Colombia: ICONTEC, 2006.

[6] F.M. Gatta, A. Geri, S. Lauria, M. Maccioni, "Backflashover simulation of HV transmission lines with enhanced counterpoise groundings", Electric Power System Research, vol. 79, no. 7, pp. 1076-1084, 2009, doi: 10.1016/j.epsr.2009.01.008

[7] W. A. Chisholm, W. Janischewskyj, "Lightning surge response of ground electrodes", IEEE Transactions on Power Delivery, vol. 9, no. 4, pp. 95-95, 1989, doi: 10.1109/MPER.1989.4310635

[8] "Guide for Improving the Lightning Performance of Transmission Lines", en IEEE Std 1243-1997, pp.144, 16 dec. 1997, doi: 10.1109/IEEESTD.1997.84660

[9] IEEE working group report, "Estimating lightning performance of transmission lines II - Updates to analytical models", IEEE Transactions on Power Delivery, vol.8, no.3, pp. 1254-1267, 1993.

[10] J. A. Martinez, F. Castro-Aranda, "Lightning performance of transmission lines using the EMTP", en IEEE Power engineering society general meeting (IEEE Cat. No 03CH37491), Toronto, Ontario, Canadá, 2003, pp. 295-300, doi: 10.1109 / PES.2003.1267186

[11] I. M. Dudurych, T. J. Gallagher, J. Corbett, M. V. Escudero, "EMTP analysis of the lightning performance of a HV transmission line", en IEE Proceedings Generation, Transmission and Distribution, vol. 150, no. 4, pp. 501-506, 2003, doi: 10.1049/ip-gtd:20030252

[12] M. Z. A. Ab. Kadir, I. Cotton, "Implementation of the modified leader progression model in backflashover analysis", en IEEE International Power and Energy Conference, Putra Jaya, Malasia, 2006, pp. 516-521, doi: 10.1109 / PECON.2006.346706 
[13] H. Swalehe, P. V. Chombo, B. Marungsri, "Flashover and back-flashover analysis with lightning strokes of $69 \mathrm{kV}$ and $24 \mathrm{kV}$ lines in Thailand using ATP/EMTP”, International Journal of Energy Conversion., vol. 6, no. 4, pp. 111-120, 2018.

[14] M. A. Abd-Allah, T. Elyan, "Analysis for Egyptian $500 \mathrm{kV}$ and $220 \mathrm{kV}$ Transmission towers", International Journal of Scientific and Research Publications, vol. 6, no. 4, pp. 289-297, 2016.

[15] I. L. Avila, G.F. di Mauro, J. A. Suárez, "Estudio de los efectos de las descargas atmosféricas sobre la confiabilidad de las líneas aéreas de transporte de energía", en III Congreso De las Américas de Distribución Eléctrica, Ciudad de Córdoba, Argentina, 2016.

[16] A. Halim, A. Bakar, H. Mokhlis, S. A. Halim, S. Sarah, M. Isa, H. A. Illias, "Analysis of Arrester Energy for $132 \mathrm{kV}$ Overhead Transmission Line due to Back Flashover and Shielding Failure", en IEEE International Conference on Power and Energy (PECon), Kota Kinabalu, 2012, pp. 683-688, doi: 10.1109/PECon.2012.6450302

[17] B. Marungsri, S. Boonpoke, A. Rawangpai, A. Oonsivilai, C. Kritayakornupong, "Study of Tower Grounding Resistance Effected Back Flashover to 500 $\mathrm{kV}$ Transmission Line in Thailand by using ATP/EMTP", International Journal of Electrical and Electronics Engineering, vol. 3, no. 10, pp. 617-624, 2009.

[18] F.M. Gatta, A. Geri, S. Lauria, "Backflashover simulation of HV transmission lines with concentrated tower grounding", Electric Power System Research, vol. 73, no. 3, pp. 373-381, 2005, doi: 10.1016/j.epsr.2004.08.011

[19] F. M. Gatta, A. Geri, S. Lauria, M. Maccioni, "Backflashover simulation of $\mathrm{HV}$ transmission lines with enhanced counterpoise groundings", Electric Power System Research, vol. 79, no. 7, pp. 1076-1084, 2009, doi: 10.1016/j.epsr.2009.01.008

[20] S. Visacro, F. H. Silveira, "Lightning Performance of Transmission Lines: Requirements of Tower-Footing Electrodes Consisting of Long Counterpoise Wires", IEEE Transactions on Power Delivery, vol. 31, no. 4, pp. 1524-1532, 2016, doi: 10.1109/TPWRD.2015.2494520
[21] J. Montaña, "Recommendations for grounding systems in lightning protection systems", Ingeniería $e$ Investigación, vol. 31, no. 2, pp. 5-10, 2011.

[22] R. Shariatinasab, J. Gholinezhad, "The effect of grounding system modeling on lightning-related studies of transmission lines", Journal of Applied Research Technolology, vol. 15, no. 6, pp. 545-554, 2017.

[23] M.A. Sargent, M. Darveniza, "Tower surge impedance", IEEE Transactions On Power Apparatus and Systems, vol. 88, no. 5, pp. 680-687, 1969, doi: 10.1109 / TPAS.1969.292357

[24] H. W. Dommel, "Electromagnetic Transients Program Reference Manual. EMTP Theory Book", en Prepared for Bonneville Power Administration, Portland, OR, USA, 1986.

[25] Ministerio de Minas y Energía, Reglamento Técnico de Instalaciones Eléctricas, 2013.

[26] M. Darveniza, F. Popolansky, E. R. Whitehead, "Lightning protection of UHV transmission lines", Electra, no. 41, pp. 39-69, 1975. 